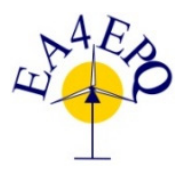

International Conference on Renewable Energies and Power Quality (ICREPQ'18)

Salamanca (Spain), 21 $1^{\text {th }}$ to $23^{\text {th }}$ March, 2018

Renewable Energy and Pourer Quality. Tournal (RE\&PQJ)

ISSN 2172-038 X, No.16 April 2018

\title{
Impact of flat-top voltage waveform distortion on harmonic current emission and summation of electronic household appliances
}

\author{
Ana Maria Blanco ${ }^{1}$, Manish Gupta ${ }^{2}$, Aurora Gil de Castro ${ }^{3}$, Sarah Rönnberg ${ }^{4}$ and Jan Meyer ${ }^{1}$ \\ ${ }^{1}$ Institute for Electrical Power Systems and High Voltage Engineering \\ Technische Universität Dresden, Germany \\ ana.blanco@tu-dresden.de, jan.meyer@tu-dresden.de \\ ${ }^{2}$ Electrical Engineering Department \\ Malaviya National Institute of Technology, Jaipur, India \\ ${ }^{3}$ Dept. of Electronic and Computer Engineering \\ Universidad de Cordoba, Spain \\ ${ }^{4}$ Luleå University of Technology, \\ Skellefteå, Sweden
}

\begin{abstract}
Electronic household appliances are non-linear loads and emit harmonics into the low voltage networks. Usually, these loads are simply modelled by constant current source models, which only represent the harmonic emission of the appliances for a single supply voltage distortion, mostly sinusoidal conditions.

Measurements have shown that the harmonic currents emitted by electronic devices can significantly depend on the circuit topology and the existing supply voltage distortion. This paper studies the impact of supply voltage distortion, which can be typically found in public low voltage networks (so-called flat-top voltage waveform), on the harmonic current emission of individual devices with different circuit topologies as well as the impact of supply voltage distortion on the harmonic summation of these devices.
\end{abstract}

\section{Key words}

Harmonic current emission, electronic household appliances, voltage distortion, attenuation effect, harmonic summation.

\section{Introduction}

The use of power electronic devices such as laptops, TVs, compact fluorescent lamps (CFL's), LED lamps and electrical vehicle chargers is continuously increasing in residential networks because of their high efficiency, smooth performance and low cost. However, these devices are nonlinear loads which inject harmonic currents into the network. The impact of individual devices to the total voltage distortion is usually negligible, but the aggregated impact of hundreds of electronic devices could considerably affect the harmonic voltages in the network.
The electronic household devices have different circuit topologies with different harmonic current emission. Most of the studies which analyze the impact of the different topologies on the power quality are based on simulations which use an ideal current source to represent these loads (e.g. [1-2]). The parameters of the current source (magnitude and phase angle of the harmonic currents) are usually obtained from measurements of the respective devices based on the specifications of international standards, such as IEC 61000-3-2 [3]. These standards define a nearly undistorted voltage for the measurement of harmonic currents.

However, in low voltage networks usually a distorted voltage with THD (Total harmonic distortion) values between $1 \%$ and $5 \%$ can be found. The voltage distortion is mainly determined by the large amount of single-phase rectifiers, which results in a flattened top that has been observed in many measurements by the authors [4]. Previous studies have shown that the voltage distortion can considerably affect the harmonic currents emitted by the electronic loads. This effect depends on the voltage waveform characteristics (magnitude and phase angle of the harmonics) [5]. Therefore, the current source may not be an adequate model for these type of loads.

The main aim of this paper is to analyze the effect of different flat-top supply voltage distortion on the harmonic current emission of a representative selection of household electronic devices with different topologies. Moreover, the impact of different levels of supply voltage distortion on 


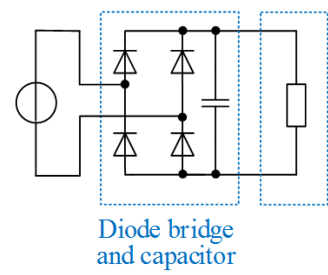

a) No-PFC

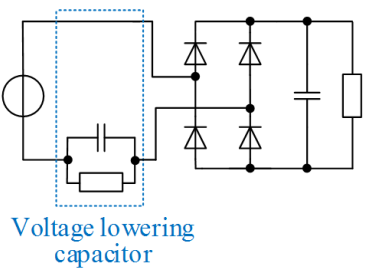

b) No-PFC (Capacitive divider)

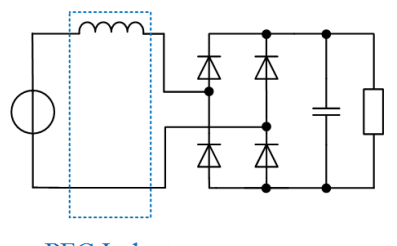

PFC Inductor

c) Passive PFC

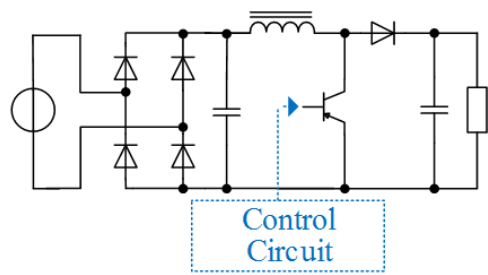

d) Active PFC

Fig. 1. Simplified circuit diagrams of the different circuit topologies used in most electronic household devices.

the summation of the harmonic currents of the selected household devices is studied in order to identify whether the harmonic summation characteristic depends also on the supply voltage distortion.

The first part of the paper gives an overview of the characteristics of the household electronic appliances and the reason of their sensitivity to the voltage distortion. The second part presents the measurement setup and discusses the results of the measurements. Furthermore, the sensitivity of each of the selected appliances to different levels and types of flat-top voltage is evaluated. The third part analyses the effect of flat-top voltage waveform on the summation of harmonic currents in mixtures of the appliances. Finally some conclusions and recommendations are given.

\section{Theoretical background}

Electronic household devices have different circuit topologies, which can be broadly classified into three categories depending on the type of power factor correction (PFC):

1- No-PFC topology: Usually consists of a simple power supply (PS) with diode bridge rectifier and smoothing capacitor. The PS provides smooth voltage to the DC load. Usually the power factor is low $(P F<0.6)$.

2- Passive-PFC topology: PS with additional series capacitors or inductors before or after the bridge diode rectifier to increase the power factor (usually $0.6 \leq P F \leq 0.9$ ).

3- Active-PFC topology: PS with advanced control circuits that shape the input current waveform. This topology has the best performance related to power factor $(P F>0.9)$.

Fig.1 shows exemplarily some simplified circuit diagrams of different circuit topologies. The main component of all the topologies is the diode-bridge rectifier with the smoothing capacitor. The capacitive divider of Fig. $1 \mathrm{~b}$ is a special case of the no-PFC topology, where a capacitor is used to lower the input voltage, but it does not improve the power factor $(P F<0.6$ for capacitive dividers $)$.

The previous classification is not the most appropriate with respect to the harmonic emission. In most of the cases, the devices with no-PFC topology produce the highest distortion ((THDi>80\%), except for the capacitive divider $(T H D i<30 \%)$. Usually, passive-PFC devices have a medium distortion $(40 \% \leq T H D i \leq 80 \%)$, and active-PFC devices have the lowest distortion $(T H D i<40 \%)$.

Real circuits contain other components, like highfrequency filters, protection elements and switching stages after the smoothing capacitor (i.e. switch mode power supply), but those elements do not have a significant impact on the low-frequency harmonic emission of the device.

As example, Fig. 2a shows the current waveforms of some devices with different circuit topologies when a sinusoidal voltage is applied. The first two signals correspond to devices with No-PFC topologies; the first curve is the typical waveform of CFLs and LED lamps, while the second curve is the typical waveform of battery chargers. Capacitive dividers are usually found in small LEDs. The passive and active PFC topologies are usually found in devices with high input power, like computers, photovoltaic inverters, electric vehicles, etc. It is important to highlight that devices of the same topology do not have exactly the same current waveform or harmonic emission due to slight differences in the circuit elements or circuit schematic implemented by the different manufacturers.

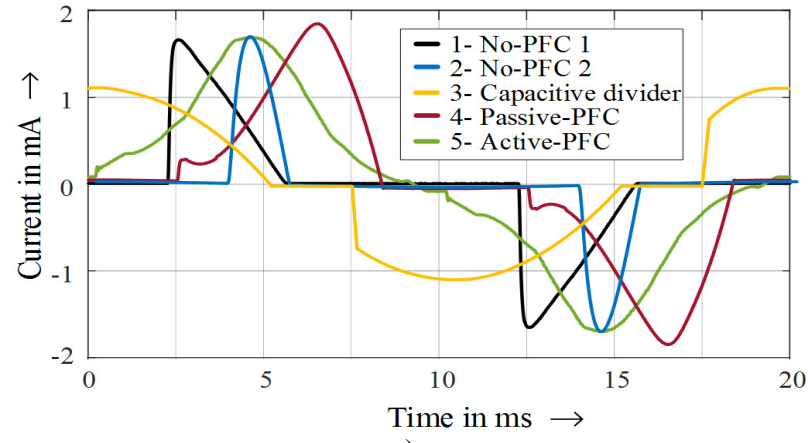

a)

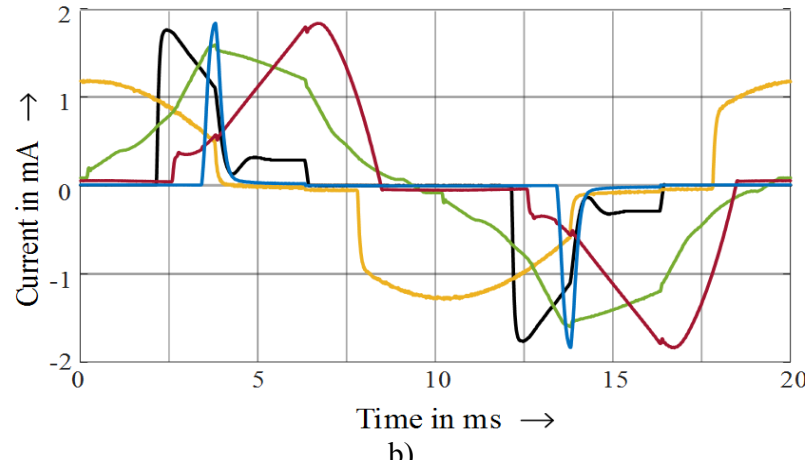

b)

Fig. 2 Current waveforms of typical electronic appliances when a sinusoidal (a) and a flat-top voltage (b) are applied. 

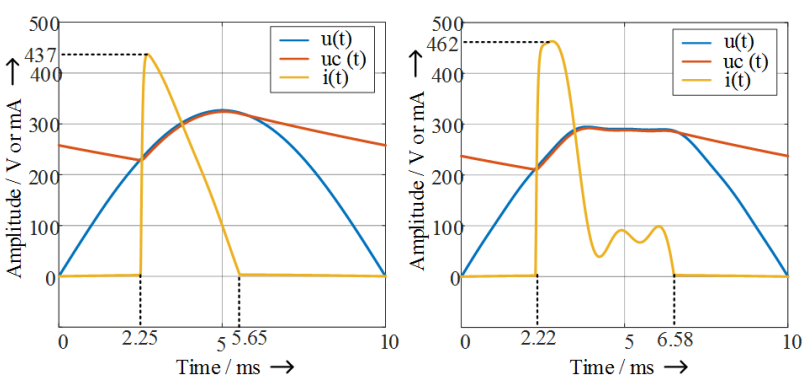

Fig.3 Simulation results of a No-PFC topology supplied with sinus (left) and flat-top (right) voltage.

The harmonic current emission of the electronic devices varies with the distortion of the supply voltage. Fig. $2 b$ shows the current emitted by the same devices of Fig.2a when a flat-top supply voltage is applied. A comparison of Fig. $2 \mathrm{a}$ and Fig. $2 \mathrm{~b}$ reveals that the harmonic current varies more or less significantly with the voltage distortion, particularly devices 1,2 , and 5 had the most significant variation. This phenomenon is usually known in the literature as attenuation effect because in most of the cases, when a flat-top voltage is applied, the harmonic emission of the electronic devices decreases, i.e. it is attenuated. However, as already proved by several authors [5-7], depending on the voltage waveform characteristics (magnitude and phase angle of the voltage harmonics), harmonic current emission of electronic devices can increase or decrease.

Changes in the input current are mainly caused by the operation characteristic of the DC-link capacitor. Fig. 3 shows the input voltage $u(t)$, input current $i(t)$ and the voltage of the DC-link capacitor uc(t) of a no-PFC topology obtained from simulations using a sinusoidal and a flat-top voltage waveforms (details about simulation parameters in [5]). Comparing both results, it is clear that the starting time and the duration of the charging state of the capacitor depends on the input voltage characteristics, which will have a direct influence on the resulting input current of the device. Moreover, oscillations on the voltage during the operation of the capacitor will also be reflected in the input current.

\section{Measurement framework}

The measurement setup consists of a programmable voltage source of $15 \mathrm{kVA}$ and a transient recorder with a sampling rate of $100 \mathrm{kS} / \mathrm{s}$ for the measurement of voltage and current waveforms, as shown in Fig. 4. The voltage and current waveforms were later processed with a FFT (acc [8], 10cycles, rectangular window) to obtain the voltage and current harmonic magnitudes and phase angles.

Electronic appliances with different circuit topologies were selected for the analysis. Table 1 lists the different appliances and shows the THDi and the total power factor measured when the devices are supplied with an undistorted sinusoidal voltage waveform. LED 1 is the only device with a capacitive divider. Depending on usage behavior, the power consumption of a PC can vary in large ranges. To include this fact in the analysis, 2 load levels $(95 \mathrm{~W}$ and $200 \mathrm{~W}$ ) were considered. The first corresponds to typical

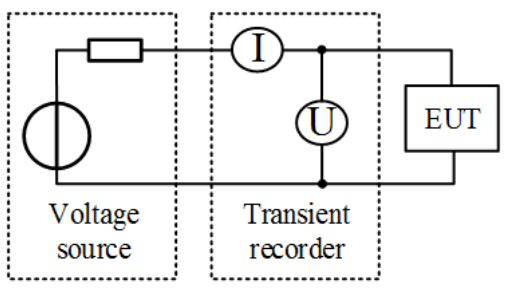

Fig. 4. Measurement setup

Table I. - Selected electronic appliances

\begin{tabular}{|c|c|c|c|c|c|}
\hline$N r$. & Topology & Device & Power $(W)$ & $P F$ & THDi (\%) \\
\hline 1 & \multirow{4}{*}{ No PFC } & CFL 1 & 20 & 0.61 & 103.7 \\
\hline 2 & & LED 1 & 1 & 0.22 & 22.9 \\
\hline 3 & & LED 2 & 8 & 0.69 & 68.1 \\
\hline 4 & & Laptop 1 & 60 & 0.43 & 203 \\
\hline 5 & \multirow{2}{*}{ Passive PFC } & \multirow{2}{*}{ PC 1} & 95 & 0.79 & 75 \\
\hline 6 & & & 200 & 0.81 & 62.6 \\
\hline 7 & \multirow{4}{*}{ Active PFC } & CFL 2 & 30 & 0.97 & 18.5 \\
\hline 8 & & Laptop 2 & 95 & 0.92 & 31.4 \\
\hline 9 & & \multirow{2}{*}{ PC 2} & 95 & 0.91 & 33 \\
\hline 10 & & & 200 & 0.93 & 33 \\
\hline
\end{tabular}

Distortion:

\begin{tabular}{|l|l|l|}
\hline Low & Medium & High \\
\hline
\end{tabular}

office applications (e.g. editing documents or browsing the internet), and the second is dedicated to more power consuming applications, like games or video rendering.

In order to obtain a comprehensive overview of the influence of the flat-top voltage on the harmonic current emission, different flat-top voltage waveforms were defined. Fig. 5 presents the different voltage waveforms, classified in three groups: Group 1 has approximately horizontal flat-top waveforms, group 2 has left-skewed flat-top waveforms, and group 3 has right-skewed flat-top waveforms. In all the cases, the $T H D u$ is below $5 \%$, which satisfies the specification of the standard IEC 61000-2-2 for voltage distortion in low-voltage networks [9]. The more pronounced the flat-top is, the higher is the voltage distortion. It should be noted that the flat-top waveform is always a mix of multiple odd harmonics with different magnitudes and phase angles. Consequently, this study will take into account the typical interaction between harmonics of different orders.

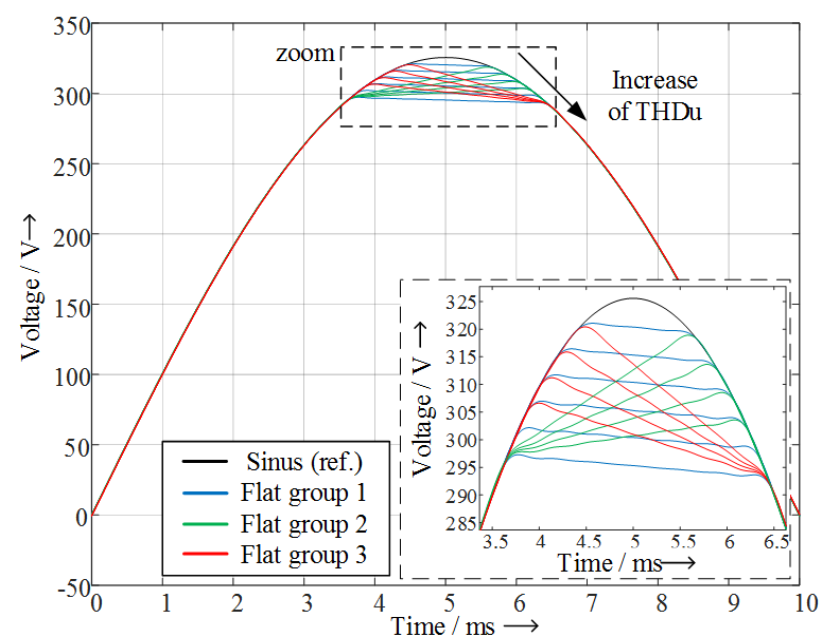

Fig. 5. Defined flat-top voltage waveforms 


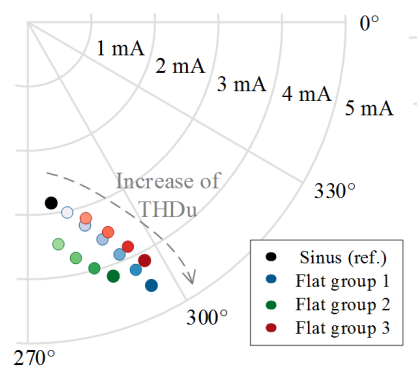

a) LED 1

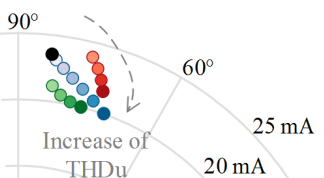
$15 \mathrm{~mA} \quad 30^{\circ}$ $10 \mathrm{~mA}$ $5 \mathrm{~mA}$.

b) LED 2

Fig. 6 Variation of the 3rd harmonic current for two LED lamps

\section{Results of individual appliances}

Fig. 6 shows exemplary the magnitude and phase angle variation of the $3^{\text {rd }}$ harmonic currents of LED 1 and LED 2 with the different flat-top voltage waveforms. The change in the colors from light to dark indicates the direction in which the voltage distortion increases, i.e. the flat-top gets more pronounced (c.f. Fig. 5). The behavior of each of the lamps is different, suggesting that also the summation of both lamps depends on the voltage distortion. While $3^{\text {rd }}$ harmonic current increases for the LED1, the same harmonic decreases for LED 2, which can be explained by the different circuit components. In both cases, there is a
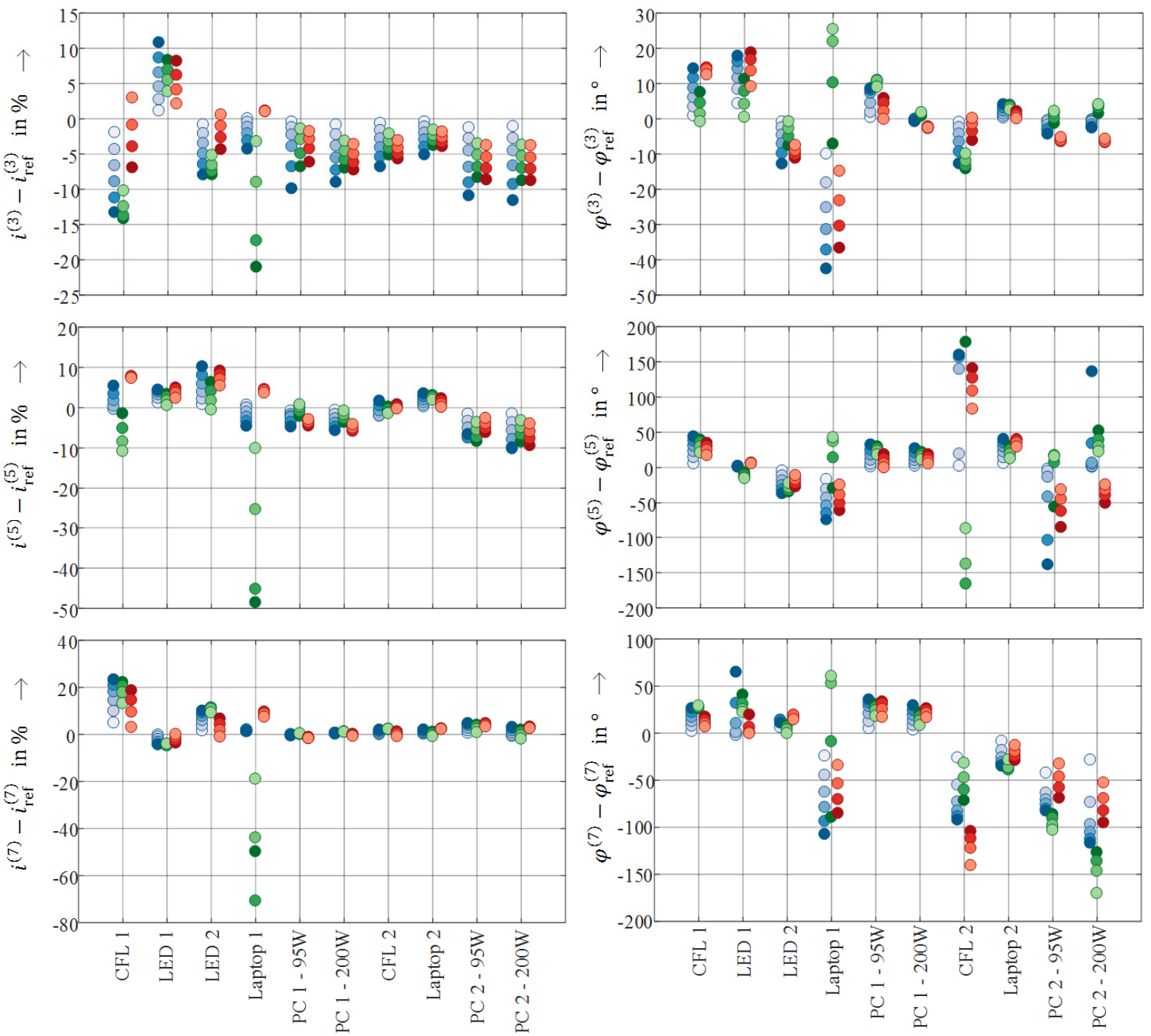

Fig.7 Variation of harmonic magnitudes and phase angles of the third, fifth and seventh harmonic with respect to the reference case. 
increase of voltage distortion (attenuation). For example, the third harmonic decreases, while the seventh harmonic current increases in most of the cases. In general, the variation of harmonic magnitudes for the No-PFC topologies is about $10 \%$ (except for laptop 1 with variations between 20 and 40\%), while for passive and active PFC topologies the variation is less than 5\% (except for the third harmonic with variations of about $10 \%$ ).

There is also a significant change in the phase angles, which increases with the harmonic order. Contrary to the harmonic magnitudes, the harmonic phase angles seems to be more sensitive for active-PFC topologies and the laptop 1 . Variations between $30^{\circ}$ and $100^{\circ}$ are observed in most of the cases and for most harmonics.

\section{Results of mixture of devices}

The presence of different devices with different topologies at one connection point can cause a diversity of harmonic phase angles and subsequently may lead to a lower magnitude of phasor sum than the arithmetical sum of the harmonic currents [5]. This is known as cancellation effect and has a high influence on the total harmonic distortion emitted by larger groups of electronic devices into the network. The cancellation effect is quantified in this paper with the harmonic cancellation factor $(\mathrm{HCF})$ individual for each harmonic:

$$
H C F^{(h)}=1-\frac{\left|\sum_{i=1}^{n} \underline{I}_{i}^{(h)}\right|}{\sum_{i=1}^{n}\left|\underline{I}_{i}^{(h)}\right|}
$$

where $\underline{\boldsymbol{I}}_{i}^{(\boldsymbol{h})}$ represents the harmonic current phasor of the device $i, n$ is the number of devices and $h$ is the order of the harmonic. $H C F$ varies between 1 (perfect cancellation between harmonic phasors) and 0 (no cancellation).

Using the measured harmonic currents of the individual devices, the $H C F$ of different mixtures of devices was calculated. Table II indicates the composition of each mixture (Device Nr. defined in Table I), and Fig. 8 shows the $H C F$ of the third and fifth harmonics obtained for each mixture and for each voltage distortion. The $H C F$ of the third harmonic does not have a high variation with the different flat-top voltages, but for the other harmonic orders,

Table II. - Mixtures of devices

\begin{tabular}{|c|c|c|c|c|c|c|}
\hline Mixture & 1 & 2 & 3 & 4 & 5 & 6 \\
\hline Devices Nr. & $1,2,3,7$ & $1-4$ & $5-10$ & $1,3,6,10$ & $3,7,4,8$ & All \\
\hline
\end{tabular}
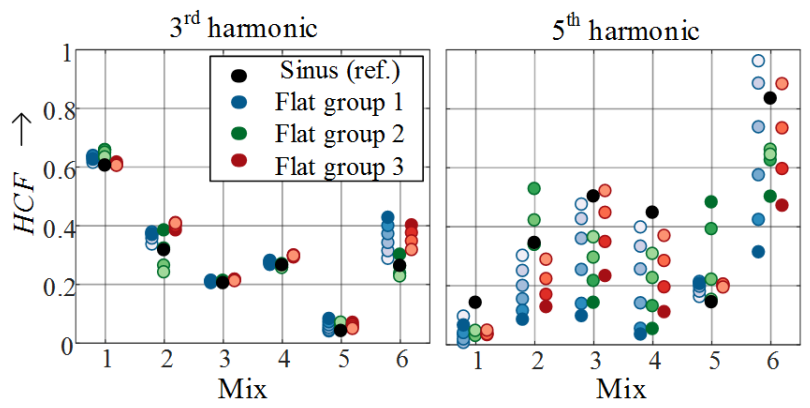

Fig. 8 Cancellation factor of different mixtures of devices as the fifth harmonic in the figure, the $H C F$ varies widely. In most of the cases, the $H C F$ decreases with the voltage distortion, indicating that, the cancellation of harmonics worsen with the increase of the voltage distortion. The cancellation of harmonics depends mainly on the type of devices in the mixture, but the results of Fig. 8 also show that the cancellation of harmonics also depends on the voltage distortion. Therefore, an accurate and reliable study of the cancellation effect of harmonic currents should take voltage distortion into account.

\section{Conclusion}

Electronic household devices have different circuit topologies, which can be broadly grouped into three categories based on the type of power factor correction method: no-PFC, passive PFC and active PFC. All topologies emit harmonic currents, which depend strongly on the distortion of the supply voltage.

Measurements showed that the no-PFC topologies are more sensitive to the voltage distortion. The variation of harmonic currents with the voltage distortion is mainly linked to the operation characteristic of the DC-link capacitor of the rectifier.

The relation between the variation of voltage harmonic magnitudes and phase angles with the corresponding changes in the harmonic currents is not linear, and difficult to characterize. Therefore, the simple current source model is not recommended to represent the harmonic emission of electronic devices in harmonic studies.

The cancelation effect of harmonic currents of order 5 and higher is highly affected by the presence of voltage harmonics. Therefore, realistic harmonic studies about the impact of electronic equipment on the harmonic studies should also consider the voltage distortion. Moreover, revision of the respective standards should be considered, because most of them take only into account the harmonic current emission under ideal sinusoidal voltage conditions.

\section{References}

[1] Koch, A., Myrzik, J., Wiesner, T., and Jendernalik, L. Harmonics and resonances in the low voltage grid caused by Compact Fluorescent Lamps. 14th International Conference on Harmonics and Quality of Power-ICHQP. Bergamo, 2010

[2] Korovesis, P., Vokas, G., Gonos, I. and Topalis, F. Influence of large-scale lamps on the line voltage distortion of a weak network supplied by photovoltaic station. IEEE Transactions on Power Delivery, Vol. 19, No. 4, October 2004. P. 1787 -1793.

[3] Electromagnetic compatibility (EMC) - Part 3-2: Limits Limits for harmonic current emissions (equipment input current $\leq 16$ A per phase), IEC Standard 61000-3-2, Mar. 2009.

[4] S. Yanchenko and J. Meyer, "Harmonic emission of household devices in presence of typical voltage distortions," in 2015 IEEE Eindhoven PowerTech, 2015, pp. 1-6.

[5] Blanco, A.M.; Yanchenko, S.; Meyer, J.; Schegner, P. Impact of supply voltage distortion on the current harmonic emission of non-linear loads. DYNA, Vol. 82, No. 192, p. 150-159, 2015. 
[6] S. Cobben, W. Kling, and J. Myrzik. "The making and purpose of harmonic fingerprints." In: 19th International Conference on Electricity Distribution - CIRED. Viena, Austria: CIRED, 2007, pp. $1-4$.

[7] Gil-de-Castro, A., Medina-Gracia, R., Ronnberg, S.K., Blanco, A., Meyer, J. "Differences in the performance between CFL and LED lamps under different voltage distortions". International Conference on Harmonics and Power Quality -ICHQP 2018 (accepted for publication).

[8] Electromagnetic compatibility (EMC) - Part 4-7: Testing and measurement techniques - General guide on harmonics and interharmonics measurements and instrumentation, for power supply systems and equipment connected thereto, IEC Standard 61000-4-7:2002+A1:2008

[9] Electromagnetic compatibility (EMC) - Part 2-2: Environment - Compatibility levels for low-frequency conducted disturbances and signalling in public low-voltage power supply systems, IEC Standard 61000-2-2:2002+A1:2016-10 